\title{
In-situ and Post-Mortem Observations of Microstructural Evolution in NiTi Polycrystals Strained by Load-biased Thermal Cycling
}

\author{
M. Bowers ${ }^{1}$, L. Yang $^{1}$, N.T. Nuhfer ${ }^{2}$, M. De Graef ${ }^{2}$, and M.J. Mills ${ }^{1}$ \\ 1. Department of Materials Science and Engineering, The Ohio State University, \\ Columbus, $\mathrm{OH} 43201$ \\ 2. Department of Materials Science and Engineering, Carnegie Mellon University, \\ Pittsburgh, PA 15213
}

Shape memory alloys (SMAs) such as NiTi offer potential for use in a wide array of applications, such as surgical stents and solid-state actuators[1]. The extensive potential of SMAs is attributed to the shape memory effect (SME) and pseudoelasticity. These shape-recovery behaviors are enabled by a thermoelastic martensite transformation, which can be induced upon heating (SME) or by application of external load (pseudoelasticity). Unfortunately, these materials are limited in practice by rapid degradation of the transformation behavior upon cyclic heating or loading. It is well established that the transformation is always accompanied by the generation of defect content [2-4], however the mechanism of formation as well as the role of these defects in subsequent transformation cycles is still a subject of research. The complex interaction between plasticity and the martensite transformation necessitates a thorough study of thermomechanical test modes and the resultant functional fatigue characteristics.

Combined thermomechanical testing, such as load-biased thermal cycling, is a critical test mode that has been explored due to its relevance to many potential applications and because it combines the effects of temperature and applied load. In an effort to better understand the coupling between matrix plasticity and transformation behavior, this study combines load-biased thermal cycling with in-situ heating STEM analysis of the austenite/martensite microstructural evolution for a number of test conditions. Additionally, STEM-based orientation mapping via a Nanomegas ASTAR system is used to investigate crystal rotation and texture development. A fully annealed 49at.\%NiTi alloy was thermally cycled between $30^{\circ} \mathrm{C}$ and $165^{\circ} \mathrm{C}$ for a range of cycles with a $150 \mathrm{MPa}$ constant tensile load and negligible load for comparison. FIB foils were taken from a gage cross-section of each specimen and heated through the transformation to austenite in a 200kV FEI Tecnai F20 S/TEM.

Fig. 1 shows the strain accumulation as a function of cycles for the load-biased condition. There is a large increase in strain with cycling for this case, while the no-load condition demonstrates only negligible strain after 100 cycles. In addition, the load-biased case shows a significant decrease in transformation strain (a narrowing hysteresis loop signifies decreased work output), where the transformation strain of the no-load sample appears to be consistent cycle to cycle. It is clear that the constraint imposed by this modest stress has a dramatic effect on the cyclic evolution of the material. Fig. 2 shows the high temperature dislocation substructures for the two extreme conditions. The 2cycle specimen has a homogeneous dislocation distribution while the 100-cycle specimen shows dense pockets of dislocations adjacent to regions with little or no visible 
diffraction contrast. Orientation mapping (see Fig. 2 insets) has indicated that this phenomenon may be due to refinement of the austenite matrix into smaller grains of varied orientation. This finding challenges the conventional belief that the martensite will always return to the same austenite structure, and suggests that accommodation may be achieved through multiple mechanisms.

The support of the National Science Foundation, Division of Materials Research under the GOALI program DMR-1207494 is gratefully acknowledged by MB, LY and MJM.

\section{References}

[1] M. Wagner et al., Materialwissenschaft Und Werkstofftechnik, 35 (2004), 320-325.

[2] R. Hamilton et al., Acta Materialia, 52 (2004) 3383-3402.

[3] D. M. Norfleet et al., Acta Materialia, 57 (2009), 3549-3561.

[4] T. Simon et al., Acta Materialia, 58 (2010), 1850-1860.

[5] This research was supported by the National Science Foundation under award \#DMR0907561.
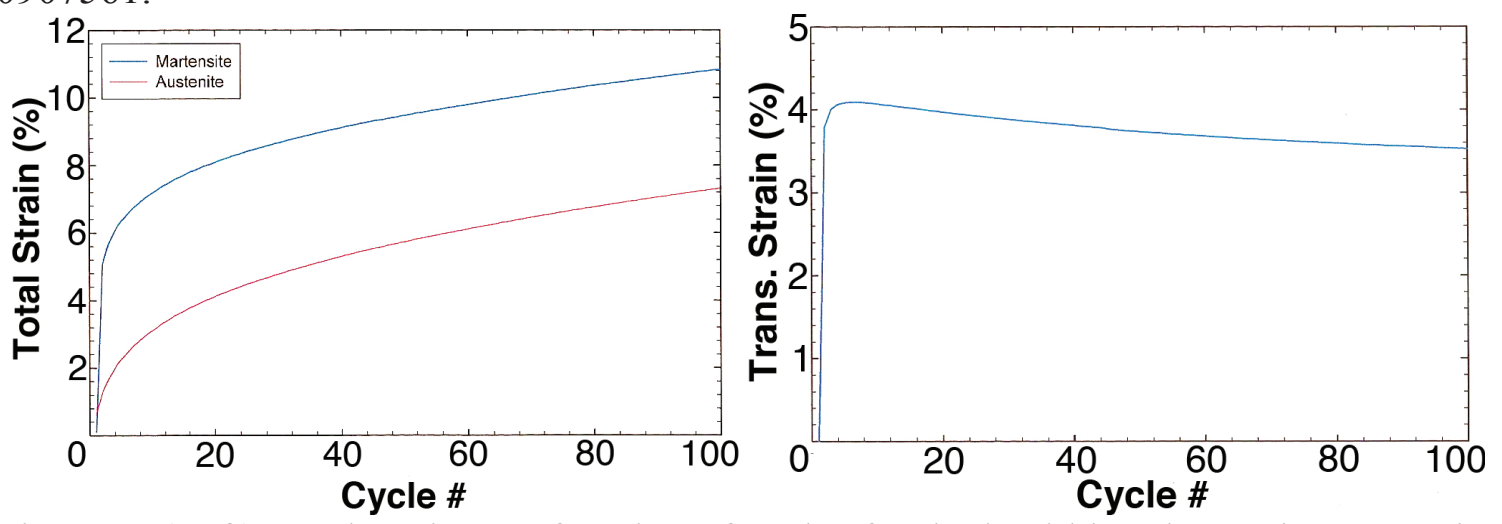

Figure 1: (Left) Total strain as a function of cycles for the load-biased case, in martensite (blue) and austenite (red). (Right) Transformation strain as a function of cycles.

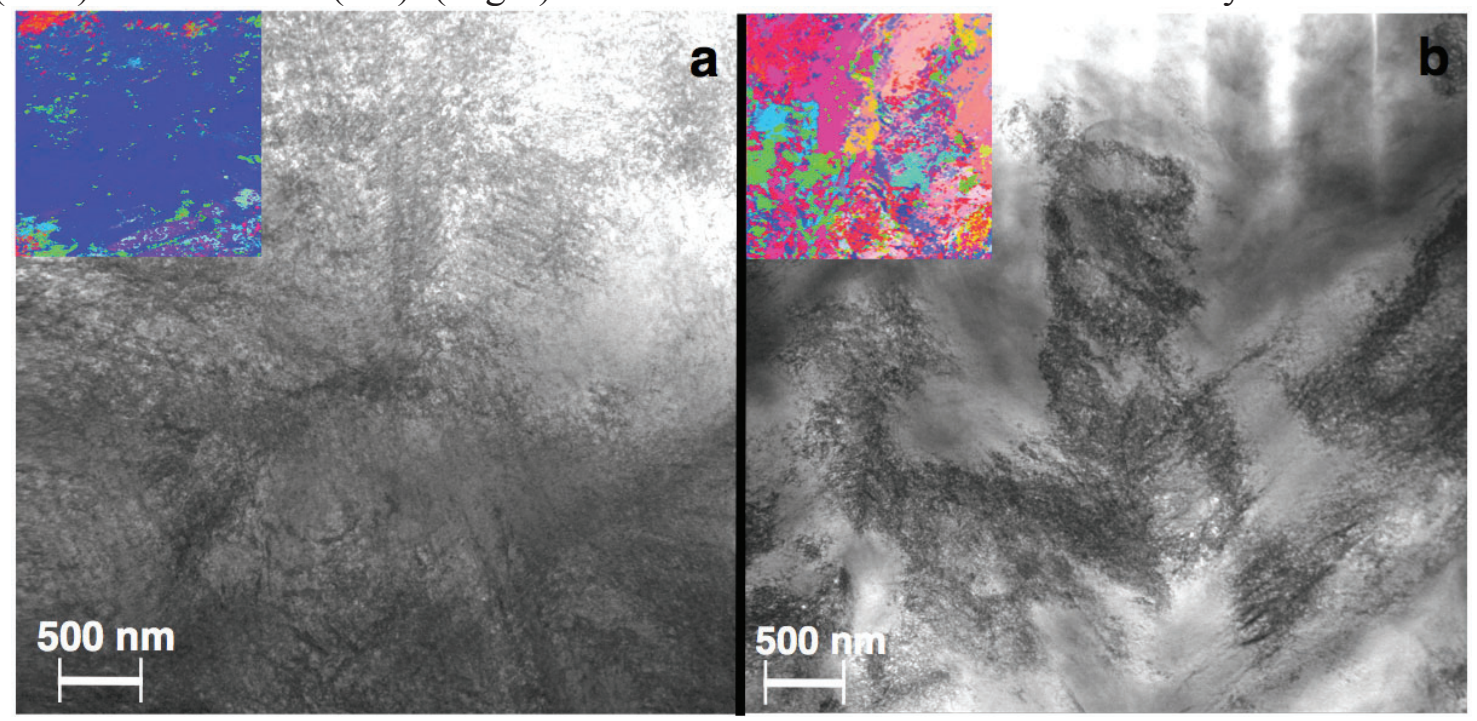

Figure 2: BF STEM micrographs taken at high temperature $\left(\sim 160^{\circ} \mathrm{C}\right)$ for (a) a 2-cycle no-load specimen and (b) a 100-cycle, $150 \mathrm{MPa}$ load-biased specimen. Insets are orientation maps from the same specimens. 